\title{
QUANTUM DISSIPATION AND QUANTUM GROUPS
}

\author{
Alfredo Iorio and Giuseppe Vitiello \\ Dipartimento di Fisica - Università di Salerno and INFN-Napoli, I84100 Salerno, Italy
}

\begin{abstract}
We discuss the rôle of quantum deformation of Weyl-Heisenberg algebra in dissipative systems and finite temperature systems. We express the time evolution generator of the damped harmonic oscillator and the generator of thermal Bogolubov transformations in terms of operators of the quantum Weyl-Heisenberg algebra. The quantum parameter acts as a label for the unitarily inequivalent representations of the canonical commutation relations in which the space of the states splits in the infinite volume limit.
\end{abstract}

PACS 02.20.+b; 03.65.Fd; 11.30.Qc;

E-mail: vitiello@sa.infn.it

Annals of Physics (N.Y.), in press. 


\section{Introduction}

The attention which has been devoted to quantum deformations ${ }^{[1,2]}$ of Lie algebras in the last years is motivated by their appearance in many problems of great physical interest, in statistical mechanics as well as in nonlinear dynamical systems, in conformal theories as well as in solid state physics. Recently, it has been also recognized ${ }^{[3]}$ that quantum deformations appear whenever a discrete (space or time) length characterizes the system under study.

Our aim in the present paper is to explicitly express the time evolution generator of the damped harmonic oscillator and the generator of the thermal Bogolubov transformations in terms of operators of the quantum deformation of the WeylHeisenberg algebra. We thus establish the relation of quantum Weyl-Heisenberg algebra with the hamiltonian of the damped harmonic oscillator and with thermal field theories.

One aspect of physical interest which emerges from our study relies in the fact that the quantum deformation parameter turns out to be related with time parameter in the case of damped harmonic oscillator and with temperature in the case of thermal field theory. In both cases, the quantum parameter acts as a label for the unitarily inequivalent representations of the canonical commutation relations in which the space of the states splits in the infinite volume limit (in the case of damped harmonic oscillator the unitarily inequivalent representations are spanned by the time evolution operator, in the case of finite temperature field theory they are spanned by the generator of the Bogolubov transformations). Such a conclusion confirms a general analysis ${ }^{[4]}$ which shows that the Weyl representations in Quantum Mechanics and the unitarily inequivalent representations in Quantum Field Theory are indeed labelled by the deformation parameter of the quantum Weyl-Heisenberg algebra. In some sense the present results, providing explicit physical examples, confirm and clarify the rôle of quantum deformations in specific applications as dissipative systems and finite temperature systems.

In refs. 5-8 some aspects of dissipation in Quantum Field Theory have been studied by considering the canonical quantization of damped harmonic oscillator and it has been proven that the space of the physical states splits into unitarily inequivalent representations of the canonical commutation relations in the infinite volume limit. Also, it has been realized that canonical quantization of the damped harmonic oscillator leads to $S U(1,1)$ time-dependent coherent states, which are well known in high energy physics as well as in quantum optics and thermal field theories. Moreover, dissipation phenomena and squeezed ${ }^{[9]}$ coherent states have been mathematically related, thus showing their common physical features ${ }^{[5]}$. In 
Sec. 2 we will summarize the formalism for the canonical quantization of the damped harmonic oscillator.

Quantum deformations of Lie algebras are well studied mathematical structures and therefore their properties need not to be presented and discussed again in this paper; we only recall that they are deformations of the enveloping algebras of Lie algebras and have Hopf algebra structure. In particular the quantum Weyl-Heisenberg algebra has the properties of graded Hopf algebra ${ }^{[10]}$. In Sec. 3 we will present the realization of quantum Weyl-Heisenberg algebra in terms of finite difference operators over the set of the entire analytic functions ${ }^{[3]}$. In this work we do not study the rôle of the coproduct operation neither we investigate the superalgebra features of quantum Weyl-Heisenberg algebra in connection with our physical examples. We plan to study such topics in the future.

Finally, in Sec. 4, the formal relation between the damped harmonic oscillator Hamiltonian and the quantum Weyl-Heisenberg algebra is established. The relation of quantum Weyl-Heisenberg algebra with thermal field theory at real time (thermo field dynamics ${ }^{[11,12]}$ ) is also shown in Sec. 4. Sec. 5 is devoted to the conclusions. A preliminary discussion on the subject of the present paper has been presented in ref. 13 .

\section{The canonical quantization of the damped harmonic oscillator}

The Feshbach and Tikochinsky ${ }^{[14]}$ quantum hamiltonian is

$$
H=\hbar \Omega\left(\alpha^{\dagger} \beta+\alpha \beta^{\dagger}\right)-\frac{i \hbar \gamma}{4 m}\left[\left(\alpha^{2}-\alpha^{\dagger 2}\right)-\left(\beta^{2}-\beta^{\dagger 2}\right)\right]
$$

where

$$
\begin{gathered}
\alpha \equiv\left(\frac{1}{2 \hbar \Omega}\right)^{\frac{1}{2}}\left(\frac{p_{x}}{\sqrt{m}}-i \sqrt{m} \Omega x\right) \quad, \quad \alpha^{\dagger} \equiv\left(\frac{1}{2 \hbar \Omega}\right)^{\frac{1}{2}}\left(\frac{p_{x}}{\sqrt{m}}+i \sqrt{m} \Omega x\right) \\
\beta \equiv\left(\frac{1}{2 \hbar \Omega}\right)^{\frac{1}{2}}\left(\frac{p_{y}}{\sqrt{m}}-i \sqrt{m} \Omega y\right) \quad, \quad \beta^{\dagger} \equiv\left(\frac{1}{2 \hbar \Omega}\right)^{\frac{1}{2}}\left(\frac{p_{y}}{\sqrt{m}}+i \sqrt{m} \Omega y\right) \\
{\left[\alpha, \alpha^{\dagger}\right]=1=\left[\beta, \beta^{\dagger}\right] \quad, \quad[\alpha, \beta]=0=\left[\alpha, \beta^{\dagger}\right] .}
\end{gathered}
$$

Of course, $\left[x, p_{x}\right]=i \hbar=\left[y, p_{y}\right], \quad[x, y]=0=\left[p_{x}, p_{y}\right] ; \Omega$ is the frequency. The modes $\alpha$ and $\beta$ refer to the damped oscillator with classical motion equation:

$$
m \ddot{x}+\gamma \dot{x}+\kappa x=0
$$


and to its time-reversed image:

$$
m \ddot{y}-\gamma \dot{y}+\kappa y=0
$$

respectively. As discussed in ref. 14 and refs. 5-8, the introduction of the $\beta$ (or $y$ ) oscillator is required in order to set up the canonical quantization scheme. In ref. 8 it has been shown that, at quantum level, the $\beta$ modes may allow quantum noise effects arising from the imaginary part of the action. By using the canonical linear transformations $A \equiv \frac{1}{\sqrt{2}}(\alpha+\beta)$ and $B \equiv \frac{1}{\sqrt{2}}(\alpha-\beta), H$ may also be written as

$$
\begin{gathered}
H=H_{0}+H_{I}, \\
H_{0}=\hbar \Omega\left(A^{\dagger} A-B^{\dagger} B\right) \quad, \quad H_{I}=i \hbar \Gamma\left(A^{\dagger} B^{\dagger}-A B\right),
\end{gathered}
$$

where the decay constant for the classical variable $x(t)$ is denoted by $\Gamma \equiv \frac{\gamma}{2 m}$.

We note that the states generated by $B^{\dagger}$ represent the sink where the energy dissipated by the quantum damped oscillator flows: the $B$-oscillator represents the (single-mode) reservoir or heat bath coupled to the $A$-oscillator.

The dynamical group structure associated with the system of coupled quantum oscillators is that of $S U(1,1)$. We also observe that $\left[H_{0}, H_{I}\right]=0$.

The time-evolution of the vacuum $|0>\equiv| n_{A}=0, n_{B}=0>, A \mid 0>=0=$ $B \mid 0>$, is controlled by $H_{I}$

$$
\begin{aligned}
& \mid 0(t)>= \exp \left(-i t \frac{H}{\hbar}\right)\left|0>=\exp \left(-i t \frac{H_{I}}{\hbar}\right)\right| 0> \\
&= \frac{1}{\cosh (\Gamma t)} \exp \left(\tanh (\Gamma t) A^{\dagger} B^{\dagger}\right) \mid 0>, \\
&<0(t) \mid 0(t)>=1 \quad \forall t \\
& \lim _{t \rightarrow \infty}<0(t) \mid 0>\propto \lim _{t \rightarrow \infty} \exp (-t \Gamma)=0 .
\end{aligned}
$$

Eq. (2.9) express the instability (decay) of the vacuum under the evolution operator $\exp \left(-i t \frac{H_{I}}{\hbar}\right)$. We note that $\mid 0(t)>$ is a two-mode Glauber coherent state ${ }^{[15,16]}$.

Time evolution transformations for creation and annihilation operators are

$$
\begin{aligned}
& \alpha \mapsto \alpha(t)=\mathrm{e}^{-i \frac{t}{\hbar} H_{I}} \alpha \mathrm{e}^{i \frac{t}{\hbar} H_{I}}=\alpha \cosh (\Gamma t)-\alpha^{\dagger} \sinh (\Gamma t), \\
& \beta \mapsto \beta(t)=\mathrm{e}^{-i \frac{t}{\hbar} H_{I}} \beta \mathrm{e}^{i \frac{t}{\hbar} H_{I}}=\beta \cosh (\Gamma t)+\beta^{\dagger} \sinh (\Gamma t)
\end{aligned}
$$

and h.c., and the corresponding ones for $A(t), B(t)$ and h.c.. We note that (2.10) are canonical transformations preserving the canonical commutation relations (2.3). 
The signs of $\sinh (\Gamma t)$ in (2.10a) and (2.10b) show that $\alpha$ and $\beta$ are each other time-reversed. From eq. (2.1) we see that the time-evolution operator $\mathcal{U}(t)$ may be written as

$$
\mathcal{U}(t)=\exp \left(-i t \frac{H_{I}}{\hbar}\right)=\exp \left(-\frac{\Gamma t}{2}\left(\alpha^{2}-\alpha^{\dagger 2}\right)\right) \exp \left(\frac{\Gamma t}{2}\left(\beta^{2}-\beta^{\dagger 2}\right)\right) .
$$

In ref. 7 it has been shown that the proper way to perform the canonical quantization of the damped harmonic oscillator is to work in the framework of Quantum Field Theory . For many degrees of freedom $\mathcal{U}(t)$ is formally (at finite volume) given by

$$
\begin{array}{r}
\mathcal{U}(t)=\prod_{\kappa} \exp \left(-\frac{\Gamma_{\kappa} t}{2}\left(\alpha_{\kappa}^{2}-\alpha_{\kappa}^{\dagger 2}\right)\right) \exp \left(\frac{\Gamma_{\kappa} t}{2}\left(\beta_{\kappa}^{2}-\beta_{\kappa}^{\dagger 2}\right)\right) \\
=\prod_{\kappa} \exp \left(\Gamma_{\kappa} t\left(A_{\kappa}^{\dagger} B_{\kappa}^{\dagger}-A_{\kappa} B_{\kappa}\right)\right) .
\end{array}
$$

We still have $\left[H_{0}, H_{I}\right]=0$ and the ground state is (at finite volume $V$ )

$$
\left|0(t)>=\prod_{\kappa} \frac{1}{\cosh \left(\Gamma_{\kappa} t\right)} \exp \left(\tanh \left(\Gamma_{\kappa} t\right) A_{\kappa}^{\dagger} B_{\kappa}^{\dagger}\right)\right| 0>\quad,
$$

with $<0(t) \mid 0(t)>=1, \quad \forall t$. Using the continuous limit relation $\sum_{\kappa} \mapsto \frac{V}{(2 \pi)^{3}} \int d^{3} \kappa$, in the infinite-volume limit we have (for $\int d^{3} \kappa \Gamma_{\kappa}$ finite and positive)

$$
\begin{gathered}
<0(t) \mid 0>\rightarrow 0 \text { as } V \rightarrow \infty \quad \forall t, \\
<0(t) \mid 0\left(t^{\prime}\right)>\rightarrow 0 \text { as } V \rightarrow \infty \quad \forall t \text { and } t^{\prime}, \quad t^{\prime} \neq t .
\end{gathered}
$$

At each time $t$ a bona fide representation $\{\mid 0(t)>\}$ of the canonical commutation relations is defined and turns out to be unitarily inequivalent to any other representation $\left\{\mid 0\left(t^{\prime}\right)>, \forall t^{\prime} \neq t\right\}$ in the infinite volume limit. In such a way the quantum damped harmonic oscillator evolves in time through unitarily inequivalent representations of canonical commutation relations (tunneling). As usual one works at finite volume and only at the end of the computations the limit $V \rightarrow \infty$ is performed.

Finally, in refs. 6 and 7 it has been shown that the representation $\{\mid 0(t)>\}$ is equivalent to the thermo field dynamics representation $\{\mid 0(\beta(t)>, \quad \beta(t) \equiv$ $1 / K T(t)\}$, thus recognizing the relation between the damped harmonic oscillator states and the finite temperature states. 
In particular, one may introduce the free energy functional for the $A$-modes

$$
\mathcal{F}_{A} \equiv<0(t)\left|\left(H_{A}-\frac{1}{\beta} S_{A}\right)\right| 0(t)>\quad,
$$

where $H_{A}$ is the part of $H_{0}$ relative to $A$ - modes only, namely $H_{A}=\sum_{\kappa} \hbar \Omega_{\kappa} A_{\kappa}^{\dagger} A_{\kappa}$, and the entropy $S_{A}$ is given by

$$
S_{A} \equiv-\sum_{\kappa}\left\{A_{\kappa}^{\dagger} A_{\kappa} \ln \sinh ^{2}\left(\Gamma_{\kappa} t\right)-A_{\kappa} A_{\kappa}^{\dagger} \ln \cosh ^{2}\left(\Gamma_{\kappa} t\right)\right\} .
$$

One then considers the stability condition $\frac{\partial \mathcal{F}_{A}}{\partial \vartheta_{\kappa}}=0 \quad \forall \kappa \quad, \vartheta_{\kappa} \equiv \Gamma_{\kappa} t$ to be satisfied in each representation, and using the definition $E_{\kappa} \equiv \hbar \Omega_{\kappa}$, one finds

$$
\mathcal{N}_{A_{\kappa}}(t)=\sinh ^{2}\left(\Gamma_{\kappa} t\right)=\frac{1}{\mathrm{e}^{\beta(t) E_{\kappa}}-1},
$$

which is the Bose distribution for $A_{\kappa}$ at time $t .\{\mid 0(t)>\}$ is thus recognized ${ }^{[7]}$ to be a representation of the canonical commutation relations at finite temperature, equivalent to the thermo field dynamics representation $\{\mid 0(\beta)>\}^{[11,12]}$.

\section{The quantum deformation of the Weyl-Heisenberg algebra}

We now introduce the realization of quantum Weyl-Heisenberg algebra in terms of finite difference operators ${ }^{[3]}$ in order to establish a formal relation with the damped harmonic oscillator hamiltonian.

Since we want to preserve the analytic properties of Lie algebra in the deformation procedure, we adopt as a framework the Fock-Bargmann representation in Quantum Mechanics ${ }^{[15]}$ (we observe that, by working in the Fock-Bargmann representation, quantum Weyl-Heisenberg algebra is incorporated into the theory of the (entire) analytic functions, a result which is by itself interesting ${ }^{[3]}$ ).

In the Fock-Bargmann representation the operators

$$
N \rightarrow z \frac{d}{d z}, \quad a^{\dagger} \rightarrow z, \quad a \rightarrow \frac{d}{d z}, \quad z \in \mathbb{C},
$$

provide a realization of the Weyl-Heisenberg algebra

$$
\left[a, a^{\dagger}\right]=\mathbb{I}, \quad[N, a]=-a, \quad\left[N, a^{\dagger}\right]=a^{\dagger} .
$$

The Hilbert space $\mathcal{F}$ is identified with the space of the entire analytic functions and has well defined inner product. The wave functions $\psi(z)$ are expressed as

$$
\psi(z)=\sum_{n=0}^{\infty} c_{n} u_{n}(z), \quad u_{n}(z)=\frac{z^{n}}{\sqrt{n !}}, \quad n \in \mathcal{I}_{+} .
$$


The set $\left\{u_{n}(z)\right\}$ provides an orthonormal basis in $\mathcal{F}$. Eq. (3.3) provides the most general representation of an entire analytic function. The conjugation of the operators introduced in eq. (3.1) is defined with respect to the inner product in $\mathcal{F}[15]$.

Next we consider the finite difference operator $\mathcal{D}_{q}$ defined by:

$$
\mathcal{D}_{q} f(z)=\frac{f(q z)-f(z)}{(q-1) z}=\frac{q^{z \frac{d}{d z}}-1}{(q-1) z} f(z)
$$

with $f \in \mathcal{F}, q=e^{\zeta}, \zeta \in \mathbb{C} . \mathcal{D}_{q}$ is the well known quantum derivative operator and, for $q \rightarrow 1$ (i.e. $\zeta \rightarrow 0$ ), it reduces to the standard derivative.

We can write the algebra

$$
\left[\mathcal{D}_{q}, z\right]=q^{z \frac{d}{d z}}, \quad\left[z \frac{d}{d z}, \mathcal{D}_{q}\right]=-\mathcal{D}_{q}, \quad\left[z \frac{d}{d z}, z\right]=z
$$

which is recognized to be the quantum deformation of the Weyl-Heisenberg algebra. In fact, let us introduce the following operators in the space $\mathcal{F}$ :

$$
N \rightarrow z \frac{d}{d z}, \quad \hat{a}_{q} \rightarrow z, \quad a_{q} \rightarrow \mathcal{D}_{q}
$$

where $\hat{a}_{q}=\hat{a}_{q=1}=a^{\dagger}$ and $\lim _{q \rightarrow 1} a_{q}=a$. The quantum Weyl-Heisenberg algebra, in terms of the operators $\left\{a_{q}, \hat{a}_{q}, N \equiv N_{q} ; q \in \mathbb{C}\right\}$, is then realized by the relations:

$$
\left[N, a_{q}\right]=-a_{q},\left[N, \hat{a}_{q}\right]=\hat{a}_{q},\left[a_{q}, \hat{a}_{q}\right] \equiv a_{q} \hat{a}_{q}-\hat{a}_{q} a_{q}=q^{N}
$$

By introducing $\quad \bar{a}_{q} \equiv \hat{a}_{q} q^{-N / 2}$, it assumes the more familiar form ${ }^{[2]}$

$$
\left[N, a_{q}\right]=-a_{q} \quad, \quad\left[N, \bar{a}_{q}\right]=\bar{a}_{q}, a_{q} \bar{a}_{q}-q^{-\frac{1}{2}} \bar{a}_{q} a_{q}=q^{\frac{1}{2} N} .
$$

The finite difference operator algebra (3.7) thus provides a realization of the quantum Weyl-Heisenberg algebra in the Fock-Bargmann representation.

We can show that the commutator $\left[a_{q}, \hat{a}_{q}\right]$ acts in $\mathcal{F}$ as follows ${ }^{[3]}$

$$
\left[a_{q}, \hat{a}_{q}\right] f(z)=q^{z \frac{d}{d z}} f(z)=f(q z) .
$$

The quantum deformation of the Weyl-Heisenberg algebra is thus strictly related with the finite difference operator $\mathcal{D}_{q}(q \neq 1)$. This suggests to us that the quantum deformation of the operator algebra should arise whenever we are in the presence of lattice or discrete structure ${ }^{[3]}$. In the following we show that this 
indeed happens in the case of damping where the finite life-time $\tau=\frac{1}{\Gamma}$ acts as the time-unit for the system.

\section{Dissipation, finite temperature and quantum Weyl-Heisenberg alge- bra}

In this section we finally express the time evolution generator of damped harmonic oscillator and the generator of the finite temperature Bogolubov transformations in terms of the commutator $\left[a_{q}, \hat{a}_{q}\right]$ of the quantum Weyl-Heisenberg algebra.

In the Fock-Bargmann representation Hilbert space $\mathcal{F}$ we have the identity

$$
2 z \frac{d}{d z} f(z)=-\left(\tilde{a}^{2}-\tilde{a}^{\dagger 2}\right) f(z)-f(z), \quad f \in \mathcal{F}
$$

with

$$
\tilde{a} \equiv \frac{1}{\sqrt{2 \hbar \Omega}}\left(\frac{p_{z}}{\sqrt{m}}-i \sqrt{m} \Omega z\right), \quad \tilde{a}^{\dagger} \equiv \frac{1}{\sqrt{2 \hbar \Omega}}\left(\frac{p_{z}}{\sqrt{m}}+i \sqrt{m} \Omega z\right), \quad\left[\tilde{a}, \tilde{a}^{\dagger}\right]=\mathbb{I}
$$

where $z \in \mathbb{C}, p_{z}=-i \hbar \frac{d}{d z}$ and $\left[z, p_{z}\right]=i \hbar$. The conjugation of $\tilde{a}$ and $\tilde{a}^{\dagger}$ is as usual well defined with respect to the inner product defined in $\mathcal{F}^{[15]}$.

We note that, by setting $\operatorname{Re}(z)=x, \tilde{a} \rightarrow \alpha$ and $\tilde{a}^{\dagger} \rightarrow \alpha^{\dagger}$ in the limit $\operatorname{Im}(z) \rightarrow$ 0 , where $\alpha$ and $\alpha^{\dagger}$ denote the annihilation and creation operators introduced in eqs. (2.2).

By putting $q=\mathrm{e}^{\theta}$ with $\theta$ real and by using eq. (4.1) we can easily check that the operator

$$
\left[a_{q}, \hat{a}_{q}\right]=\exp \left(\theta z \frac{d}{d z}\right)=\frac{1}{\sqrt{q}} \exp \left(-\frac{\theta}{2}\left(\tilde{a}^{2}-\tilde{a}^{\dagger 2}\right)\right) \equiv \frac{1}{\sqrt{q}} \hat{\mathcal{S}}(\theta)
$$

generates the Bogolubov transformations:

$$
\tilde{a}(\theta)=\hat{\mathcal{S}}(\theta) \tilde{a} \hat{\mathcal{S}}(\theta)^{-1}=\tilde{a} \cosh \theta-\tilde{a}^{\dagger} \sinh \theta
$$

and h.c.. We then observe that in the limit $\operatorname{Im}(z) \rightarrow 0$ eq. (4.4) and its h.c. give

$$
\tilde{a}(\theta) \mapsto \alpha(\theta)=\alpha \cosh \theta-\alpha^{\dagger} \sinh \theta \text { as } \operatorname{Im}(z) \rightarrow 0
$$

and h.c..

We also observe that the right hand side of $(4.3)$ is an $S U(1,1)$ group element. By defining $\frac{1}{2} \tilde{a}^{2}=K_{-}, \frac{1}{2} \tilde{a}^{\dagger 2}=K_{+}, \frac{1}{2}\left(\tilde{a}^{\dagger} \tilde{a}+\frac{1}{2}\right)=K_{3}$, we easily see they 
close the $s u(1,1)$ algebra. We remark that the transformation $(4.5)$, which is a canonical transformation, is exactly the one which relates the Weyl representations of the canonical commutation relations in Quantum Mechanics [4] and thus the deformation parameter $q=\mathrm{e}^{\theta}$ acts as a label for the Weyl representations.

Next, we introduce the quantum Weyl-Heisenberg algebra operators $b_{q^{\prime}}$ and $\hat{b}_{q^{\prime}}$ corresponding to the doubled degree of freedom $\beta$ introduced in Sec. 2 in order to set up the canonical quantization of the damped harmonic oscillator; let $\tilde{b}$ and $\tilde{b}^{\dagger}$ be defined, in a similar way to $\tilde{a}$ and $\tilde{a}^{\dagger}$, by

$$
\tilde{b} \equiv \frac{1}{\sqrt{2 \hbar \Omega}}\left(\frac{p_{\zeta}}{\sqrt{m}}-i \sqrt{m} \Omega \zeta\right), \quad \tilde{b}^{\dagger} \equiv \frac{1}{\sqrt{2 \hbar \Omega}}\left(\frac{p_{\zeta}}{\sqrt{m}}+i \sqrt{m} \Omega \zeta\right), \quad\left[\tilde{b}, \tilde{b}^{\dagger}\right]=\mathbb{I}
$$

with $\zeta \in \mathbb{C}, \operatorname{Re}(\zeta) \equiv y, p_{\zeta}=-i \hbar \frac{d}{d \zeta}$ and $\left[\zeta, p_{\zeta}\right]=i \hbar$, so that

$$
2 \zeta \frac{d}{d \zeta} f(\zeta)=-\left(\tilde{b}^{2}-\tilde{b}^{\dagger 2}\right) f(\zeta)-f(\zeta), \quad f \in \mathcal{F}
$$

and $\tilde{b} \rightarrow \beta, \tilde{b}^{\dagger} \rightarrow \beta^{\dagger}$ as $\operatorname{Im}(\zeta) \rightarrow 0$. Then we see that the operator

$$
\left[a_{q}, \hat{a}_{q}\right]\left[b_{q^{\prime}}, \hat{b}_{q^{\prime}}\right]=\exp \left(-\frac{\theta}{2}\left[\left(\tilde{a}^{2}-\tilde{a}^{\dagger 2}\right)-\left(\tilde{b}^{2}-\tilde{b}^{\dagger 2}\right)\right]\right)
$$

with $q^{\prime}=q^{-1}$, acts as the time evolution operator $\mathcal{U}(t)$ (cf. eq. (2.12)) in the $\operatorname{limits} \operatorname{Im}(z) \rightarrow 0$ and $\operatorname{Im}(\zeta) \rightarrow 0$ provided we set $q=\mathrm{e}^{\theta}$, with $\theta \equiv \Gamma t$ :

$$
\begin{aligned}
& {\left[a_{q}, \hat{a}_{q}\right]\left[b_{q^{\prime}}, \hat{b}_{q^{\prime}}\right] \mapsto \exp \left(-\frac{\Gamma t}{2}\left[\left(\alpha^{2}-\alpha^{\dagger 2}\right)-\left(\beta^{2}-\beta^{\dagger 2}\right)\right]\right)=\mathcal{U}(t)} \\
& \text { as } \operatorname{Im}(z) \rightarrow 0 \text { and } \operatorname{Im}(\zeta) \rightarrow 0 \text {. }
\end{aligned}
$$

Eq. (4.9) is the wanted expression of the time-evolution generator of the damped harmonic oscillator in terms of the quantum Weyl-Heisenberg algebra operator $\left[a_{q}, \hat{a}_{q}\right]\left[b_{q^{\prime}}, \hat{b}_{q^{\prime}}\right]$.

For many degrees of freedom, the Bogolubov transformations, corresponding to eqs. (2.10), can be implemented for every $\kappa$ as inner automorphism for the algebra $s u(1,1)_{\kappa}$. As shown in ref. 7 , at every time $t$ we have a copy $\left\{A_{\kappa}(t), A_{\kappa}^{\dagger}(t), B_{\kappa}(t), B_{\kappa}^{\dagger}(t) ;|0(t)>| \forall \kappa\right\}$ of the original algebra induced by the time evolution operator which can thus be thought of as a generator of the group of automorphisms of $\bigoplus_{\kappa} s u(1,1)_{\kappa}$ parameterized by time $t$ (we have a realization of the operator algebra at each time t, which can be implemented by Gel'fandNaimark-Segal construction in the $\mathrm{C}^{*}$-algebra formalism). Notice that the various copies become unitarily inequivalent in the infinite-volume limit, as shown by eqs. 
(2.14): the space of the states splits into unitarily inequivalent representations of the canonical commutation relations each one labelled by time parameter $t$. Our discussion can be then generalized to the case of many degrees of freedom by observing that eq. (4.9) holds for each couple of modes $\left(\alpha_{\kappa}, \beta_{\kappa}\right)$ and formally we have

$$
\prod_{\kappa}\left[a_{\kappa, q}, \hat{a}_{\kappa, q}\right]\left[b_{\kappa, q^{\prime}}, \hat{b}_{\kappa, q^{\prime}}\right] \rightarrow \exp \left(-\frac{i}{\hbar} H_{I} t\right)=\mathcal{U}(t) \quad, \quad \text { as } \operatorname{Im}\{z, \zeta\} \rightarrow 0
$$

where $q=\mathrm{e}^{\theta_{\kappa}}, \theta_{\kappa} \equiv \Gamma_{\kappa} t, q^{\prime}=q^{-1}, \operatorname{Im}\{z, \zeta\}$ denotes the imaginary part of FockBargmann representation $z$ - and $\zeta$-variables associated to each $\alpha_{\kappa}$ and $\beta_{\kappa}$ mode and $\mathcal{U}(t)$ is given by eq. (2.12). Notice that for simplicity of notation here $q$ denotes $q_{\kappa}$.

We can then conclude that, through its time dependence, the deformation parameter $q(t)$ labels the unitarily inequivalent representations $\{\mid O(t)>\}$.

On the other hand, we also observe that eq. (4.10) leads to representation of the generator of the thermal Bogolubov transformation in terms of the operators $\prod_{\kappa}\left[a_{\kappa, q}, \hat{a}_{\kappa, q}\right]\left[b_{\kappa, q^{\prime}}, \hat{b}_{\kappa, q^{\prime}}\right]$ : indeed, by resorting to the discussion of Sec. 2 where the representation $\{\mid O(t)>\}$ for the damped harmonic oscillator has been shown to be the same as the thermo field dynamics representation $\{\mid O(\beta(t))>\}$ (see refs. 6-7), we also recognize the strict relation between quantum Weyl-Heisenberg algebra and finite temperature Quantum Field Theory .

As a matter of fact, from eq. (2.17) we can set $\theta_{\kappa}(t) \equiv \theta_{\kappa}(\beta(t))$ and the

operator $\mathcal{G} \equiv \prod_{\kappa} \exp \left[\theta_{\kappa}(\beta(t))\left(A_{\kappa}^{\dagger} B_{\kappa}^{\dagger}-A_{\kappa} B_{\kappa}\right)\right]$ indeed implements the (timedependent) finite temperature Bogolubov transformation in thermo field dynamics $[7,11,12]$.

We finally remark that the derivation of eq. (4.10) (and (4.8)) holds even independently of the above discussion of the damped harmonic oscillator quantization and, by setting $\theta_{\kappa}=\theta_{\kappa}(\beta)$, we see that the operator $\prod_{\kappa}\left[a_{\kappa, q}, \hat{a}_{\kappa, q}\right]\left[b_{\kappa, q^{\prime}}, \hat{b}_{\kappa, q^{\prime}}\right]$ leads to the generator of the Bogolubov transformations in conventional thermo field dynamics in the $\operatorname{Im}\{z, \zeta\} \rightarrow 0$ limit: quantum Weyl-Heisenberg algebra thus appears as the natural candidate to study thermal field theories, too.

\section{Conclusions}

We have shown that $\prod_{\kappa}\left[a_{\kappa, q}, \hat{a}_{\kappa, q}\right]\left[b_{\kappa, q^{\prime}}, \hat{b}_{\kappa, q^{\prime}}\right]$ acts as time-evolution operator for the damped harmonic oscillator and as generator of thermal Bogolubov transformation in the $\operatorname{Im}\{z, \zeta\} \rightarrow 0$ limit. 
In the discussion presented above a crucial rôle is played by the existence of infinitely many unitarily inequivalent representations of the canonical commutation relations in Quantum Field Theory . In ref. 4 the quantum Weyl-Heisenberg algebra has been discussed in relation with the von Neumann theorem in Quantum Mechanics and it has been shown on a general ground that the quantum deformation parameter acts as a label for the Weyl systems in Quantum Mechanics and for the unitarily inequivalent representations in Quantum Field Theory; the mapping between different (i.e. labelled by different values of $q$ ) representations (or Weyl systems) being performed by the Bogolubov transformations (at finite volume). In this paper we have shown by the explicit examples of damped harmonic oscillator and finite temperature systems the physical meaning of such a labelling (further examples are provided by unstable particles in Quantum Field Theory ${ }^{[17]}$, by quantization of the matter field in curved space-time ${ }^{[18]}$, by theories with spontaneous breakdown of symmetry where different values of the order parameter are associated to different unitarily inequivalent representations (different phases)). In the case of damping, as well as in the case of time-dependent temperature, the system time-evolution is represented as tunneling through unitarily inequivalent representations: the non-unitary character of time-evolution (arrow of time) is thus expressed by the non-unitary equivalence of the representations in the infinite volume limit. It is remarkable that at the algebraic level this is made possible through the quantum deformation mechanism which organizes the representations in an ordered set by means of the labelling.

In ref. 3 it has been also shown that the commutator $\left[a_{q}, \hat{a}_{q}\right]$ acts as squeezing generator (indeed the operator $\hat{S}(\theta)$ in eq. (4.3) acts like the squeezing generator with respect to $\tilde{a}$ and $\tilde{a}^{\dagger}$ operators), a result which together with the conclusions of this paper confirms the relation between dissipation and squeezed coherent states exhibited in ref. 5. In turn, quantum groups have been also shown ${ }^{[19]}$ to be the natural candidates to study squeezed coherent states.

The above remarks strongly suggest to us that quantum deformations are mathematical structures which are characteristic of basic, deep features of Quantum Field Theory and much work still needs to be done to fully investigate the relation between Quantum Field Theory and quantum groups. For example, in this paper we have not studied the rôle played by the coproduct operation of the quantum deformation. We plan to study this subject in the future.

We are glad to acknowledge useful and enjoying discussions with E.Celeghini, S.De Martino, S.De Siena, V. Man'ko and M.Rasetti. 


\section{References}

1 Drinfeld V.G., Proc. ICM Berkeley, CA; A.M. Gleason, ed,; AMS, Providence, R.I., 1986, page 798.

Jimbo M., Int. J. of Mod. Phys. A4 (1989) 3759.

Manin Yu.I., Quantum groups and Non-Commutative Geometry, Centre de Recherches Mathématiques, Montreal, 1988.

2 L.C.Biedenharn, J.Phys. A22 (1989) L873

A.J.Macfarlane, J. Phys. A22 (1989) 4581

3 E.Celeghini, S.De Martino, S.De Siena, M.Rasetti and G. Vitiello, Mod. Phys. Lett. B 7 (1993) 1321; Quantum Groups, Coherent States, Squeezing and Lattice Quantum Mechanics, preprint 1993

4 A.Iorio and G.Vitiello, Mod. Phys. Lett. B 8 (1994) 269

5 E. Celeghini, M. Rasetti, M. Tarlini and G. Vitiello, Mod. Phys. Lett. B 3 (1989) 1213

6 E.Celeghini, M.Rasetti and G.Vitiello in Thermal Field Theories and Their Applications, H.Ezawa, T.Aritmitsu and Y.Hashimoto Eds., Elsevier, Amsterdam 1991, p. 189

7 E. Celeghini, M. Rasetti and G. Vitiello, Annals of Phys. (N.Y.) 215 (1992) 156

8 Y.N.Srivastava, G.Vitiello and A.Widom, Quantum Dissipation and quantum noise, Annals of Phys. (N.Y.), in press

9 H.P.Yuen, Phys. Rev. A13 (1976) 2226

10 E.Celeghini, T.D.Palev and M.Tarlini, Mod. Phys.Lett. B5 (1991) 187

P.P.Kulish and N.Yu.Reshetikin, Lett. Math. Phys. 18 (1989) 143

11 Y. Takahashi and H. Umezawa, Collective Phenomena 2 (1975) 55

12 H. Umezawa, H. Matsumoto and M. Tachiki, Thermo Field Dynamics and Condensed States, North-Holland Publ. Co., Amsterdam 1982

13 A.Iorio and G.Vitiello, in Banff/CAP Workshop on Thermal Field Theory, F.C. Khanna et al. eds., World Sci., Singapore 1994, p.71

14 H.Feshbach and Y.Tikochinsky, Transact. N.Y. Acad. Sci. 38 (Ser. II) (1977) 44 
15 A.Perelomov, Generalized Coherent States and Their Applications, SpringerVerlag, Berlin, Heidelberg 1986

16 J.R.Klauder and E.C.Sudarshan, Fundamentals of Quantum Optics, Benjamin, New York 1968

17 S. De Filippo and G. Vitiello, Lett. Nuovo Cimento 19 (1977) 92

18 M. Martellini, P. Sodano and G. Vitiello, Nuovo Cimento 48 A (1978) 341

19 E.Celeghini, M.Rasetti and G.Vitiello, Phys. Rev. Lett. 66 (1991), 2056 